\title{
Relay-Assisted Quantum-Key Distribution Over Long Atmospheric Channels
}

\author{
Majid Safari, Student Member, IEEE, and Murat Uysal, Senior Member, IEEE
}

\begin{abstract}
In this paper, we investigate a terrestrial relay-assisted scheme for a free-space quantum key distribution (QKD) system based on BB84 protocol. We consider the deployment of passive relays which simply redirect the qubits to the next relay node or to the receiver without performing any measurement or detection process. Based on a near-field analysis, we derive an upper bound on quantum bit error rate (QBER) of the relay-assisted QKD system. Our results demonstrate that the relay-assisted scheme is able to outperform point-to-point direct transmission for long link ranges in which turbulence effects are particularly degrading.
\end{abstract}

Index Terms-Free-space optics, quantum-key distribution, relay-assisted systems.

\section{INTRODUCTION}

C RYPTOSYSTEMS are an indispensable part of modern telecommunication networks to secure the privacy of data transmission and to protect it from electronic copying, cloning, and destroying, and to also deter unauthorized entry into the network. Vast majority of today's cryptosystems are able to offer only computational security within the limitations of conventional computing power and the realization of quantum computers would, for example, make electronic money instantly worthless. Based on the firm laws of quantum mechanics rather than some unproven foundations of mathematical complexity, quantum cryptography provides a radically different solution for key distribution promising unconditional security.

Quantum cryptography builds on a well-known quantum physics rule that no measurement can be taken without perturbing the quantum system, unless the quantum state and the measurement are compatible. It guarantees a provenly-secure communication between a transmitting party (conventionally named as Alice) and a receiving party (conventionally named as Bob) where any eavesdropping attempt by Eve can be detected by them through a public communication during error correction procedure.

An end-to-end quantum cryptosystem includes an initial phase of quantum key distribution (QKD) and a subsequent

Manuscript received January 25, 2009; revised May 10, 2009. First published June 05, 2009; current version published August 28, 2009. The work of M. Uysal was supported in part by the Natural Sciences and Engineering Research Council of Canada (NSERC) Strategic Project under Grant STPSC 356750

The authors are with the Department of Electrical and Computer Engineering, University of Waterloo, Waterloo, ON N2L3G1, Canada (e-mail: m3safari@uwaterloo.ca; muysal@ece.uwaterloo.ca).

Color versions of one or more of the figures in this paper are available online at http://ieeexplore.ieee.org.

Digital Object Identifier 10.1109/JLT.2009.2024554 one-time pad encryption process [1]. The first QKD protocol proposed by Bennett and Brassard [2] is today widely known as BB84 which uses elementary quantum systems such as polarized photons to transfer secret keys between two points. As an alternative to BB84, Ekert [3] has proposed another protocol which involves the emission of two qubits (quantum bits) from a source to Alice and Bob instead of transmitting a single qubit from Alice to Bob. Assuming that the two emitted qubits from the source are in a maximally entangled state, Ekert's protocol avoids trusting the source which can be targeted by eavesdropping attacks. Despite rather different implementation structures, both protocols guarantee equivalently secured QKD systems. A number of other protocols also exist in the literature [4], but they essentially build on these two main protocols.

In analysis, design, and optimization of QKD systems, a critical issue to consider is the operating environment. The common form of quantum channel which has been intensively studied so far is fiber optic. Although today's telecommunications networks based on optical fibers are very advanced, such channels may not always be available. A cost-effective and easy-to-install alternative to fiber optic is free-space optical (FSO) communications on which this paper focuses. With the potential high-data-rate capacity and wide bandwidth on unregulated spectrum, FSO communications has enjoyed a growing attention in recent years for classical data transmission, see, e.g., [5]-[8]. Its unique properties make it appealing for a number of applications, including last-mile access, metropolitan area network extensions, enterprise/local area network connectivity, fiber backup, back-haul for wireless cellular networks, redundant link, and disaster recovery. With the recent commercialization successes, FSO technology has reached to a certain maturity for classical data transmission and is a reliable alternative to fiber optics in quantum cryptographic applications as well. In contrast to optical fibers, the dispersion (birefringence) effects of FSO links are negligible especially at high transmission windows of atmosphere (e.g., around wavelength of $770 \mathrm{~nm}$ ). This guarantees the consistency of the propagating photon's polarization and makes FSO particularly attractive for QKD systems.

Previous studies on terrestrial free-space QKD systems have presented analytical approaches to characterize atmospheric effects [9]-[11] and demonstrated some experimental results [12]-[14]. A major disadvantage associated with free-space QKD systems is the performance degradation due to absorption, scattering, diffraction, and turbulent-induced scintillation experienced in atmospheric channels [9]-[14]. This severely limits the range of FSO QKD system.

In this paper, we propose relay-assisted transmission as a powerful method to overcome the range limitations in terrestrial 
free-space QKD systems. Relay-assisted QKD has been originally proposed in [15] for fiber optic QKD systems. In the relaying scheme of [15] called as quantum repeater, long quantum channel is divided into shorter hops in which perfect entangled pairs are created. Connecting these individual entangled pairs through entanglement swapping leads to a single entangled pair which is used for QKD based on Ekert's protocol. The quantum repeaters are able to improve the performance by overcoming the exponential path loss of signal. However, they require using quantum memory and entanglement purification to ensure creation of perfect entangled pairs in the individual hops. In [16], a simpler relaying scheme has been proposed which avoids the use of quantum memory and entanglement purification. This scheme is not anymore able to mitigate the exponential loss of signal, but it still improves the performance of fiber optic QKD systems by suppressing the aggravated effect of detector dark counts experienced in long distance links. Unlike [15], [16], a class of quantum relays called as trusted relays have been further introduced in [17], [18]. Trusted relays are assumed to be sufficiently reliable to have partial or perfect knowledge of the distributing keys. For example, a trusted quantum relay may perform the well-known intercept/resend eavesdropping strategy to direct Alice's transmitted qubits to Bob without endangering the security of the QKD system [17].

Quantum relaying has been also studied over FSO channels, but the current results are mainly restricted for satellite transmission [19], [20]. In [19], Hughes et al. have considered a relay-assisted QKD scenario for satellite communication between two terrestrial nodes without line-of-sight path to each other. A trusted relay in a satellite generates secure keys with the two nodes and then reveals the XOR of the two keys [19]. To support the idea, a feasibility analysis is also reported in [19] based on the estimation of key generation capability of QKD system between a ground station and a LEO satellite. In [20], Aspelmeyer et al. have studied a similar satellite-based QKD setup using quantum entanglement. They have investigated the link attenuation of surface/satellite-to-satellite systems to demonstrate the feasibility of their idea.

To the best of our knowledge, a detailed investigation of terrestrial relay-assisted free-space QKD systems has not been yet reported. Aiming to fill this research gap, we consider a relay-assisted system employing passive relay(s). The relays are passive in the sense that they simply redirect the qubits without any detection or quantum measurement involved. These relays, which can be implemented by adaptive optics [21]-[25], reconstruct the turbulence-degraded wave-front of the received beam and redirect the resulting collimated beam to the next relay or Bob. We investigate the efficiency of such relay-assisted systems to combat the range limitations in a terrestrial scenario. Based on a near-field analysis [9], we derive an upperbound on quantum bit error rate (QBER) as a performance criterion of free-space QKD systems and optimize it with respect to the number of relays. Our results demonstrate that, although the relay-assisted QKD scheme increases the average number of background photons collected at Bob, it is able to significantly decrease the photon loss caused by diffraction and turbulence. The proposed scheme therefore outperforms point-topoint counterparts in long ranges.
The rest of paper is organized as follows. In Section II, we introduce the relay-assisted system model and describe the QKD protocol under consideration. In Section III, we derive bounds on sift and error probabilities, and the resulting upper-bound on the QBER of the relay-assisted QKD system. In Section IV, we provide numerical results and discussions based on our derived QBER expression. Finally, we conclude in Section V.

\section{SYSTEM MODEL}

We consider a relay-assisted free-space QKD system in which the transmitted qubits by Alice travel through $K$ relay nodes before detection by Bob. Conventional amplify-and-forward and detect-and-forward relaying schemes typically deployed in wireless communications are of no use for QKD systems ${ }^{1}$. Here, we consider passive relays which simply redirect the qubits to the next relay node or to Bob without performing any measurement or detection process.

Fig. 1 illustrates a schematic diagram of a relay-assisted QKD system which uses BB84 protocol for key distribution. In this system, Alice uses a polarization basis randomly chosen from two certain polarization bases (e.g., $0^{\circ} / 90^{\circ}$ and $-45^{\circ} /+45^{\circ}$ ) for each qubit. She sends the qubit with a random bit value of 0 or 1 using polarization encoding of photons where the first polarization in each basis $\left(0^{\circ}\right.$ and $\left.-45^{\circ}\right)$ represents 0 and the second one $\left(90^{\circ}\right.$ and $\left.+45^{\circ}\right)$ represents 1 . The relay receives the transmitted optical beam and reconstructs its turbulence-degraded wave-front using adaptive optics before pointing it to the next relay or Bob. Adaptive optics involves the use of a wave-front sensor which estimates the turbulence-induced phase error information at the beginning of each block of key transmission and a deformable mirror which uses this information to correct the atmospheric distortion.

At the receiver side, Bob also chooses a random basis from the two bases using a passive beam splitter. At the outputs of the beam splitter, two polarization detection units measure the quantum state of the possibly coming photon based on the two different bases. Each of these units includes a polarizing beam splitter (PBS) to decide between two orthogonal polarization states of the corresponding basis and two single-photon Geigermode avalanche photodiodes (APDs) at the outputs of the PBS for photon count. To avoid uncorrelated measurement results due to incompatibility of the chosen bases, Alice and Bob construct the secure key only based on the qubits received at the sift events. Sift events correspond to the bit intervals in which exactly one of the APDs registers a count and both Alice and Bob have chosen the same basis. According to BB84 protocol, Alice and Bob can identify the sift events by exchanging information in a public communication channel. After identifying the sifted qubits, Alice and Bob follow a standard set of operations to identify and correct errors which have occurred in the sifted bits. These errors are caused by dark counts or background noise as well as by potential eavesdropper's intervention. Then, Alice and Bob perform a procedure called privacy amplification to prevent Eve from keeping useful information about the key

\footnotetext{
${ }^{1}$ No amplification can be performed at relay nodes without perturbing the quantum system [4]. Furthermore, since the relays are not necessarily trusted, neither measurements nor detection can be performed at the relay nodes.
} 


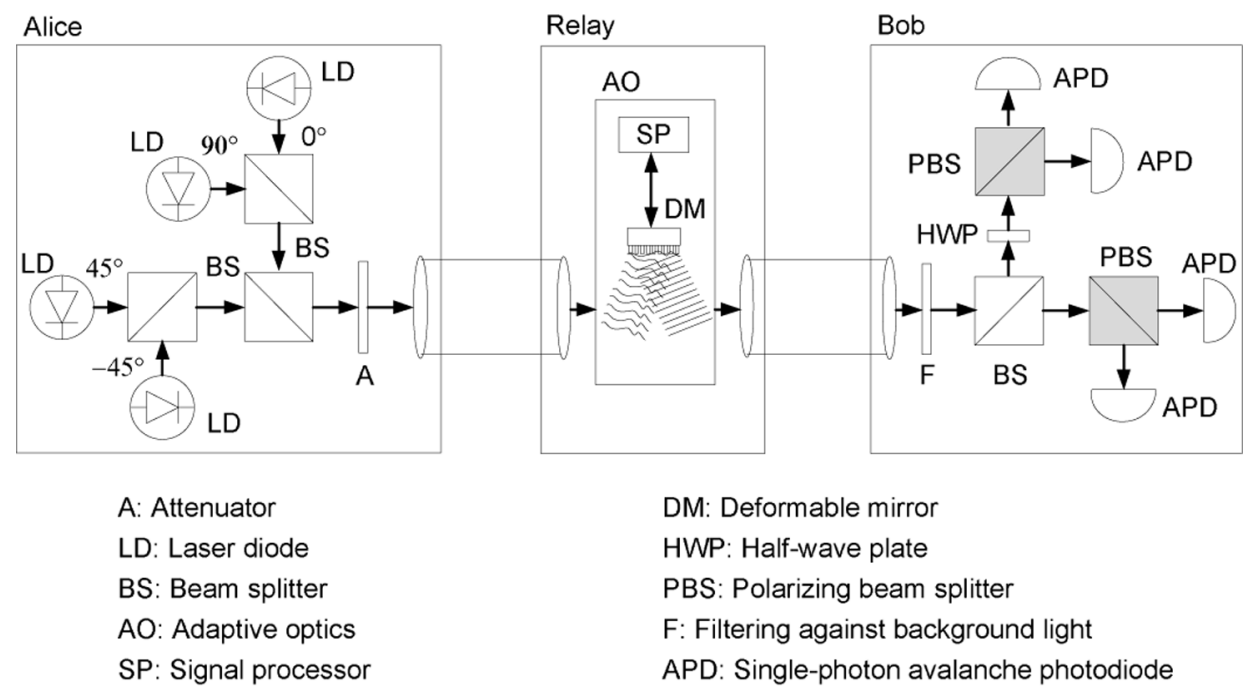

Fig. 1. Schematic diagram of the relay-assisted QKD system with a single relay $(K=1)$.

and finally establish a shared one-time pad key to use for secure communication [4]. It is worth emphasizing that any eavesdropping attempt which takes place either at the relay nodes or at any other point within the FSO link can be detected by Alice and Bob during the error correction process of the BB84 protocol.

For each qubit, Alice generates a polarized optical pulse with an average photon number of $n_{s}$ which is encoded with the corresponding polarization state of the qubit for a randomly chosen basis. Due to the atmospheric effects (i.e., diffraction, atmospheric turbulence, and absorption-and-scattering loss), the $i$ th relay node $(i=1,2, \ldots K)$ collects only a random fraction $\gamma_{i}$ of the transmitted photons in the $i$ th hop. The relay then redirects the received photons by pointing the light beam to the next relay node (or Bob) without performing any amplification/detection process. At the destination side, Bob collects a fraction $\gamma_{K+1}$ of the transmitted photons from the last relay node which corresponds to an overall fraction ${ }^{2} \gamma=\gamma_{1} \gamma_{2} \cdots \gamma_{K+1}$ of the originally transmitted photons from Alice.

In the $i$ th hop $(i=1,2, \ldots K+1)$, a spatial beam pattern $\xi_{i}(\vec{\rho})$ is assumed to be transmitted from a circular pupil $R_{i}$ with diameter $d_{i}$ located in the $z=0$ plane. ${ }^{3}$ A field pattern $\xi_{i}^{\prime}\left(\vec{\rho}^{\prime}\right)$ is collected within the pupil $R_{i}^{\prime}$ with diameter $d_{i}^{\prime}$ in the $z=L_{i}$ plane where $L_{i}$ is the length of $i$ th hop. The received field pattern by the $i$ th relay $(i=1,2, \ldots K)$ or Bob $(i=K+1)$ can be expressed as [9]

$$
\xi_{i}^{\prime}\left(\vec{\rho}^{\prime}\right)=\int_{R_{i}} \xi_{i}(\vec{\rho}) h_{i}\left(\vec{\rho}, \vec{\rho}^{\prime}\right) e^{-a L_{i} / 2} d \vec{\rho}
$$

where $a$ is the extinction coefficient which determines the loss due to absorption and scattering. In (1), $h_{i}\left(\vec{\rho}, \vec{\rho}^{\prime}\right)$ denotes the Green's function for atmospheric propagation through clear turbulent air in the $i$ th hop and is given by [9]

$$
h_{i}\left(\vec{\rho}, \vec{\rho}^{\prime}\right)=\frac{e^{j k L_{i}+j k\left|\vec{\rho}-\vec{\rho}^{\prime}\right|^{2} / 2 L_{i}}}{j \lambda L_{i}} e^{\chi\left(\vec{\rho}, \vec{\rho}^{\prime}\right)+j \phi\left(\vec{\rho}, \vec{\rho}^{\prime}\right)}
$$

${ }^{2}$ We assume negligible optics losses within the relay nodes.

${ }^{3}$ Without loss of generality, we assume that the coordinate system is properly transformed for each hop. where $\lambda$ is the wavelength and $k=2 \pi / \lambda$ is the wave number. In (2), $\chi\left(\vec{\rho}, \vec{\rho}^{\prime}\right)$ and $\phi\left(\vec{\rho}, \vec{\rho}^{\prime}\right)$ are, respectively, the stochastic log-amplitude and phase fluctuation terms describing scintillation caused by atmospheric turbulence. The strength of atmospheric turbulence is characterized by the variance of $\log$-amplitude fluctuation $\sigma_{\chi}^{2}$ which is a function of link length $L_{i}$, wave number $k$, and refractive index structure constant $C_{n}^{2}$. It is given by [26]

$$
\sigma_{\chi}^{2}(d)=0.124 k^{7 / 6} C_{n}^{2} L_{i}^{11 / 6}
$$

The fraction parameter [9] of the $i$ th hop $(i=1,2, \ldots K+1)$ is expressed in terms of transmit and receive beam patterns as

$$
\gamma_{i}=\frac{\int_{R_{i}^{\prime}}\left|\xi_{i}^{\prime}\left(\vec{\rho}^{\prime}\right)\right|^{2} d \vec{\rho}^{\prime}}{\int_{R_{i}}\left|\xi_{i}(\vec{\rho})\right|^{2} d \vec{\rho}}
$$

We assume that the relay and destination nodes collect an average number of $n_{B 0}$ background photons per polarization. Taking into account the background photons redirected by relays, the total average number of background photons collected at Bob's receiver per polarization can be expressed as 4

$$
\begin{aligned}
n_{B} & =n_{B 0}\left(1+\gamma_{K+1}+\gamma_{K} \gamma_{K+1}+\cdots+\gamma_{2} \cdots \gamma_{K+1}\right) \\
& =n_{B 0}\left[1+\sum_{i=2}^{K+1} \prod_{j=i}^{K+1} \gamma_{j}\right] .
\end{aligned}
$$

Besides the background noise, each of the single-photon detectors (APDs) at Bob's receiver registers an average number of $n_{D}$ dark counts.

${ }^{4}$ Equation (5) overestimates the effect of redirected background photons for the relay-assisted system since the associated path loss is assumed to be as low as the path loss of the transmitted collimated beam. 


\section{Performance Bounds for the Relay-Assisted FREE-SPACE QKD SYSTEM}

In this section, we investigate the performance of the relayassisted free-space QKD system through the derivation of an upper-bound on QBER. QBER is given by [4]

$$
\mathrm{QBER}=\frac{\operatorname{Pr}(\text { error })}{\operatorname{Pr}(\text { sift })}
$$

where $\operatorname{Pr}($ sift $)$ and $\operatorname{Pr}$ (error) are probabilities of sift and error, respectively. Using the results from [9], we can express the conditional sift and error probabilities of the relay-assisted QKD system (conditioned on $\mathbf{V}_{\gamma}=\left\{\gamma_{1}, \gamma_{2}, \ldots \gamma_{K+1}\right\}$ ) as

$$
\begin{aligned}
\operatorname{Pr}\left(\text { sift } \mid \mathbf{V}_{\gamma}\right)= & \eta\left(\frac{n_{S} \gamma}{2}+2 n_{N}\right) \\
& \cdot \exp \left[-\eta\left(n_{S} \gamma+4 n_{N}\right)\right] \\
\operatorname{Pr}\left(\operatorname{error} \mid \mathbf{V}_{\gamma}\right)= & \eta n_{N} \exp \left[-\eta\left(n_{S} \gamma+4 n_{N}\right)\right]
\end{aligned}
$$

where $\eta$ is the quantum efficiency of Geiger-mode APDs and $n_{N} \triangleq n_{B} / 2+n_{D}$ denotes the average number of noise counts at each of Bob's detectors.

To obtain unconditional probabilities of sift and error, we first need to statistically characterize $\mathbf{V}_{\boldsymbol{\gamma}}$. We use the singular value decomposition of $h_{i}\left(\vec{\rho}, \vec{\rho}^{\prime}\right), i=1,2, \ldots, K+1$, which is given by [9], [27]

$$
h_{i}\left(\vec{\rho}, \vec{\rho}^{\prime}\right)=\sum_{n=1}^{\infty} \sqrt{\mu_{i}^{n}} \phi_{i}^{n}\left(\vec{\rho}^{\prime}\right) \Phi_{i}^{n^{*}}(\vec{\rho})
$$

where $\mu_{i}^{n}$ 's are the eigenvalues which satisfy $1 \geq \mu_{i}^{1} \geq \mu_{i}^{2} \geq$ $\mu_{i}^{3} \geq \cdots \geq 0 .\left\{\Phi_{i}^{n}(\vec{\rho})\right\}$ and $\left\{\phi_{i}^{n}\left(\vec{\rho}^{\prime}\right)\right\}$ are, respectively, the input and output eigenfunction vectors which constitute complete orthonormal sets on $R_{i}$ and $R_{i}^{\prime}$. Let $\mu_{i}$ denote the largest eigenvalue in (9) (i.e., $\left.\mu_{i} \triangleq \mu_{i}^{1}\right)$ and $\Phi_{i}(\vec{\rho})$ denote the corresponding input eigenfunction (i.e., $\Phi_{i}(\vec{\rho}) \triangleq \Phi_{i}^{1}(\vec{\rho})$ ). Using (1), (4), and (9), an upper bound on the fraction parameter $\gamma_{i}$ can be obtained as [9]

$$
\gamma_{i} \leq \mu_{i} e^{-a L_{i}}
$$

with equality when $\overline{\xi_{i}}(\vec{\rho})=\Phi_{i}(\vec{\rho})$. Here, $\overline{\xi_{i}}(\vec{\rho})$ is the normalized transmit beam pattern of the $i$ th hop which can be expressed as

$$
\overline{\xi_{i}}(\vec{\rho})=\frac{\xi_{i}(\vec{\rho})}{\sqrt{\int_{R_{i}}\left|\xi_{i}(\vec{\rho})\right|^{2} d \vec{\rho}}} .
$$

Under atmospheric turbulence conditions which exhibit a slowly-varying nature, Alice and relay nodes can employ adaptive optics to generate the optimum transmit field pattern as

$$
\xi_{i}(\vec{\rho})=\left[\sqrt{\int_{R_{i}}\left|\xi_{i}(\vec{\rho})\right|^{2} d \vec{\rho}}\right] \Phi_{i}(\vec{\rho})
$$

which guarantees that the equality in (10) is achieved yielding the optimum fraction parameter (i.e., maximum power transfer). Let $\mu$ denote the product of the individual largest eigenvalues, i.e., $\mu=\mu_{1} \mu_{2} \cdots \mu_{K+1}(0 \leq \mu \leq 1)$. The optimum overall fraction parameter can be then written as $\gamma=\mu e^{-a L}$ where $L=L_{1}+L_{2}+\cdots+L_{K+1}$. Unfortunately, for near-field propagation, a statistical description of $\mu_{i}$ is not available in the literature. However, note that $E\left\{\mu_{i}\right\}$ is the maximum achievable average power transfer over the $i$ th hop obtained through the use of optimum beam pattern. Therefore, it can be simply lower bounded by the average power transfer of any deterministic beam pattern, such as in [9], [27], as

$$
\begin{aligned}
E\left\{\mu_{i}\right\} \geq \hat{\mu}_{i} \equiv \frac{8 \sqrt{D_{f}}}{\pi} & \int_{0}^{1} e^{-\left(D_{i}\left(d_{i} x\right) / 2\right)} J_{1}\left(4 x \sqrt{D_{f}}\right) \\
& \times\left(\cos ^{-1}(x)-x \sqrt{1-x^{2}}\right) d x
\end{aligned}
$$

where $J_{1}(\cdot)$ is the first-order Bessel function of the first kind, $D_{i}(\cdot)$ is the $i$ th hop's spherical-wave wave structure function given by $D_{i}(\nu)=1.09 k^{2} C_{n}^{2} L_{i} \nu^{5 / 3}$, and $D_{f}$ is the Fresnel number product of the $R_{i}$ and $R_{i}^{\prime}$ pupils given by $D_{f}=\left(\left(\pi d_{i} d_{i}^{\prime}\right) / 4 \lambda L_{i}\right)^{2}$.

\section{A. Bound on the Sift Probability}

Let $\mathbf{V}_{\boldsymbol{\mu}}=\left\{\mu_{1}, \mu_{2}, \ldots \mu_{K+1}\right\}$ denote the vector of the largest eigenvalues (within each hop) and define $Z\left(\mathbf{V}_{\boldsymbol{\mu}}\right)$ as a function of $\mathbf{V}_{\boldsymbol{\mu}}$ as

$$
\begin{aligned}
Z\left(\mathbf{V}_{\boldsymbol{\mu}}\right)= & \eta\left(n_{S} \gamma+4 n_{N}\right) \\
= & \eta n_{S} e^{-a L} \prod_{j=1}^{K+1} \mu_{j}+4 \eta n_{D} \\
& +2 \eta n_{B 0}\left[1+\sum_{i=2}^{K+1} \prod_{j=i}^{K+1} e^{-a L_{j}} \mu_{j}\right] .
\end{aligned}
$$

Using (14), we can rewrite (7) for the case of optimum beam transmission as

$$
\operatorname{Pr}\left(\text { sift } \mid \mathbf{V}_{\boldsymbol{\mu}}\right)=\frac{Z\left(\mathbf{V}_{\boldsymbol{\mu}}\right)}{2} e^{-Z\left(\mathbf{V}_{\boldsymbol{\mu}}\right)}=\frac{(\alpha y+\beta)}{2} e^{-(\alpha y+\beta)}
$$

where $\alpha, \beta$, and $y$ are given by 5

$$
\begin{aligned}
\alpha & =Z\left(\mathbf{V}_{\boldsymbol{\mu}}=\mathbf{1}\right)-Z\left(\mathbf{V}_{\boldsymbol{\mu}}=\mathbf{0}\right) \\
& =\eta\left[n_{S} e^{-a L}+2 n_{B 0}\left[\sum_{i=2}^{K+1} \prod_{j=i}^{K+1} e^{-a L_{j}}\right]\right] \\
\beta & =Z\left(\mathbf{V}_{\boldsymbol{\mu}}=\mathbf{0}\right)=\eta\left(2 n_{B 0}+4 n_{D}\right) \\
y & =\frac{Z\left(\mathbf{V}_{\boldsymbol{\mu}}\right)-\beta}{\alpha} .
\end{aligned}
$$

Averaging (15) over $y$, we obtain the sift probability as

$$
\operatorname{Pr}(\text { sift })=\int_{0}^{1} \frac{(\alpha y+\beta)}{2} e^{-(\alpha y+\beta)} p(y) d y
$$

${ }^{5} \mathbf{0}$ and $\mathbf{1}$ denote all-zero and all-one vectors, respectively, 
where $p(y)$ is the probability density function (pdf) of $y$. Defining $f(y) \triangleq(\alpha y / 2+\beta / 2) e^{-(\alpha y+\beta)}$ and noting that it is a concave function for $0 \leq \alpha y+\beta<2$, we can lower bound (19) as

$$
\begin{aligned}
\operatorname{Pr}(\text { sift }) & \geq \int_{0}^{1}[f(0)(1-y)+f(1) y] p(y) d y \\
& =f(0)(1-E\{y\})+f(1) E\{y\} .
\end{aligned}
$$

Using (13), (14), and (18), and the fact that the elements of $\mathbf{V}_{\boldsymbol{\gamma}}$ (and therefore the elements of $\mathbf{V}_{\boldsymbol{\mu}}$ ) are statistically independent, $E\{y\}$ can be bounded as

$$
\begin{aligned}
E\{y\} \geq \hat{y} & \triangleq \frac{n_{S} e^{-a L} \prod_{j=1}^{K+1} \hat{\mu}_{j}+2 n_{B 0}\left[\sum_{i=2}^{K+1} \prod_{j=i}^{K+1} e^{-a L_{j}} \hat{\mu}_{j}\right]}{n_{S} e^{-a L}+2 n_{B 0}\left[\sum_{i=2}^{K+1} \prod_{j=i}^{K+1} e^{-a L_{j}}\right]} .
\end{aligned}
$$

Inserting (21) in (20) and noting that $f(y)$ is increasing for $0 \leq$ $\alpha y+\beta<1$, we have a lower bound on the sift probability as

$$
\begin{aligned}
\operatorname{Pr}(\text { sift }) & \geq f(0)(1-\hat{y})+f(1) \hat{y} \\
& =\left(\frac{\beta}{2}\right) e^{-\beta}[1-\hat{y}]+\left(\frac{\alpha}{2}+\frac{\beta}{2}\right) e^{-(\alpha+\beta)} \hat{y}
\end{aligned}
$$

under the practically reasonable condition of

$$
\eta\left(n_{S} e^{-a L}+4 n_{N}\right)<1
$$

\section{B. Bound on the Error Probability}

Ignoring the effects of diffraction and turbulence on the redirected background photons (from the relays), a pessimistic noise count at each of Bob's detectors (i.e., $\hat{n}_{N}=n_{N} \mid \mathrm{v}_{\mu}=1$ ) can be written as

$$
\hat{n}_{N}=\frac{n_{B 0}}{2}\left[1+\sum_{i=2}^{K+1} \prod_{j=i}^{K+1} e^{-a L_{j}}\right]+n_{D}
$$

which obviously satisfies $\hat{n}_{N} \geq n_{N}$. Therefore, we can bound (8) as

$$
\operatorname{Pr}\left(\operatorname{error} \mid \mathbf{V}_{\boldsymbol{\gamma}}\right) \leq \eta \hat{n}_{N} e^{-\eta\left(n_{S} \gamma+4 \hat{n}_{N}\right)}
$$

Inserting $\gamma=\mu e^{-a L}$ in (24) and averaging the resulting expression over $\mu$, we have

$$
\operatorname{Pr}(\text { error }) \leq \int_{0}^{1} \eta \hat{n}_{N} e^{-\eta\left(n_{S} \mu e^{-a L}+4 \hat{n}_{N}\right)} p(\mu) d \mu
$$

where $p(\mu)$ is the pdf of $\mu$. Defining $g(\mu) \triangleq$ $\eta \hat{n}_{N} e^{-\eta\left(n_{S} \mu e^{-a L}+4 \hat{n}_{N}\right)}$ and noting that it is a convex function, we can further upper bound (25) as

$$
\operatorname{Pr}(\text { error }) \leq \int_{0}^{1}[g(0)(1-\mu)+g(1) \mu] P(\mu) d \mu
$$

$$
\begin{aligned}
& =g(0)(1-E\{\mu\})+g(1) E\{\mu\} \\
& \leq g(0)(1-\hat{\mu})+g(1) \hat{\mu}
\end{aligned}
$$

where we define $\hat{\mu}=\hat{\mu}_{1} \hat{\mu}_{2} \cdots \hat{\mu}_{K+1}$. The first bound in (26) comes from the convexity of $g(\mu)$. The last bound results from the bound in (13) assuming that $\mu_{i}$ 's are statistically independent and noting that $g(\mu)$ is a decreasing function. Expanding (26), we obtain

$$
\operatorname{Pr}(\text { error }) \leq \eta \hat{n}_{N} e^{-\eta 4 \hat{n}_{N}}[1-\hat{\mu}]+\eta \hat{n}_{N} e^{-\eta\left(n_{S} e^{-a L}+4 \hat{n}_{N}\right)} \hat{\mu} .
$$

Replacing (22) and (27) in (6), we obtain an upper bound on QBER as

$$
\mathrm{QBER} \leq \frac{2 \eta \hat{n}_{N} e^{-\eta 4 \hat{n}_{N}}\left[(1-\hat{\mu})+e^{-\eta n_{S} e^{-a L}} \hat{\mu}\right]}{\beta e^{-\beta}(1-\hat{y})+(\alpha+\beta) e^{-(\alpha+\beta)} \hat{y}} .
$$

It is important to note that, although at the beginning of this derivation we consider optimum beam shaping in (10) to achieve the equality, the derived bound in (28) is valid for non-optimized transmission. Because the lower-bound on average $\mu_{i}$ given in (13) is based on the assumption of a deterministic beam pattern rather than the optimum one [9].

Finally, as a benchmark to demonstrate the efficiency of the proposed relaying scheme in compensating the degrading effects of atmospheric turbulence, we consider a QKD system operating over non-turbulent air. The exact QBER of such a QKD system is given by [9]

$$
\mathrm{QBER}=\frac{2 n_{N}}{n_{S} \mu_{0} e^{-a L_{A B}}+4 n_{N}}
$$

where $L_{A B}$ is the length of line-of-sight link connecting Alice and $\mathrm{Bob}$ and $\mu_{0}$ is the largest eigenvalue of the singular value decomposition of vacuum-propagation Green's function given in [28].

\section{NUMERICAL RESULTS AND DISCUSSIONS}

In this section, we demonstrate the performance of relay-assisted QKD scheme under consideration and compare its performance with point-to-point direct transmission. We assume $\lambda=0.77 \mu \mathrm{m}, C_{n}^{2}=1 \times 10^{-15} \mathrm{~m}^{-2 / 3}$, and operation in clear weather conditions with visibility of $10 \mathrm{~km}$ which corresponds to the absorption and scattering loss of about $2 \mathrm{~dB} / \mathrm{km}$. Under the assumption that proper temporal, spectral, and spatial filters are employed, a constant average number of background count, $n_{B 0}=10^{-4}$ is assumed [12]. Bob's APD detectors are operated at the detection efficiency of $\eta=0.5$ with average dark count of $n_{D}=10^{-6}$ [9]. We also assume that all the receive and transmit pupils have the same diameter $d$ and the relay nodes are located equidistant along the path connecting Alice and Bob, i.e., $L_{A B}=L$.

Before we illustrate QBER performance, we study the behavior of the overall fraction of collected photons at Bob's receiver, i.e., $\gamma$. We normalize $\gamma$ by the total amount of absorption and scattering loss experienced in the direct path from Alice to Bob, i.e., $e^{-a L_{A B}}$. Based on the assumption made on the relay locations, we have $\gamma / e^{-a L_{A B}}=\mu e^{-a L} / e^{-a L_{A B}}=\mu$. Fig. 2 plots the lower bound on the average of $\mu$ (i.e., $\hat{\mu}$ ) calculated using (13) for a relay-assisted QKD system with pupil diameter 


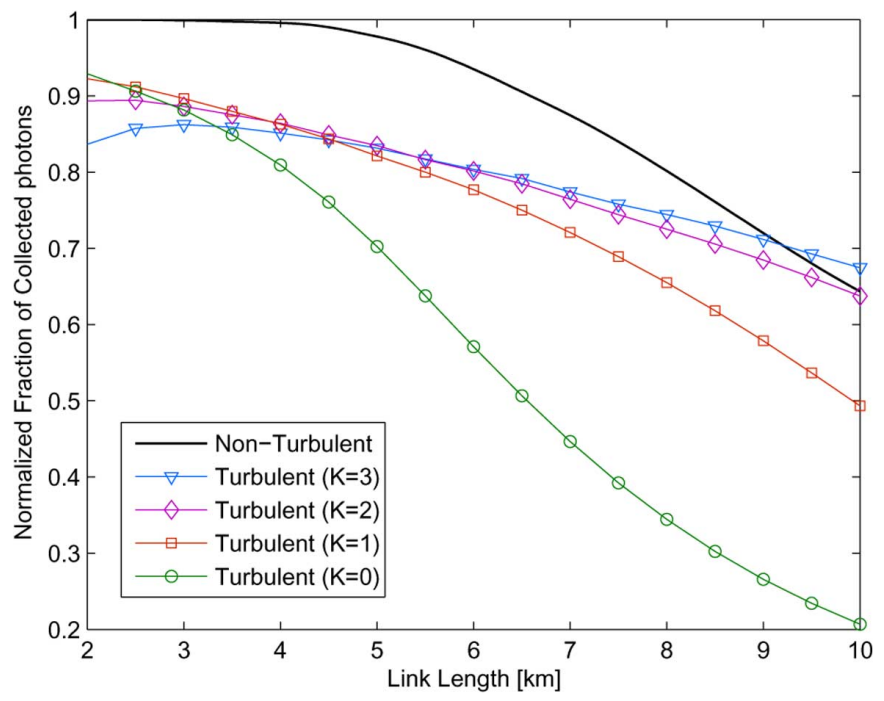

Fig. 2. Normalized fraction of collected photons at Bob's receiver in relayassisted QKD system for different number of relays $(d=10 \mathrm{~cm})$.

of $d=10 \mathrm{~cm}$ operating over turbulent atmosphere. We consider relay-assisted systems with different number of relays, i.e., $K=1,2$, and 3. The results for direct QKD systems operating over turbulent (i.e., $\hat{\mu}, K=0$ ) and non-turbulent (i.e., $\mu_{0}$ given in [28]) atmosphere are further included as benchmarks. Comparing the performance curves of over the turbulent and non-turbulent atmosphere for the direct systems in Fig. 2, we observe that atmospheric turbulence degrades the performance of QKD systems particularly over the long links where stronger turbulence effects are experienced. For example, the average number of photons collected over a $10-\mathrm{km}$ non-turbulent atmospheric path is more than three times the average number of collected photons in the corresponding turbulent case. It is obvious from Fig. 2 that, similar to the classical multi-hop FSO system [8], the relay-assisted QKD scheme takes advantage of the resulting shorter hops with weaker turbulence effects and improves the performance of the QKD system. For example, deployment of two relays would be sufficient to compensate the decrease in the average number of collected photons caused by turbulence for a $10-\mathrm{km}$ link.

Fig. 3 demonstrates the upper bound on QBER performance of the relay-assisted and direct QKD systems operating over turbulent atmosphere as a function of the link range $\left(L_{A B}=L\right)$ which are calculated through (28). We assume $d=10 \mathrm{~cm}$ and $n_{s}=1$. As a benchmark, we include the QBER performance of direct QKD system over non-turbulent atmosphere calculated through (29). It is observed from Fig. 3 that the relay-assisted scheme improves the QBER performance of the links longer than $5.5 \mathrm{~km}$. For example, for a link with the length of $10 \mathrm{~km}$, the presence of turbulence causes 193\% increase in QBER of the direct QKD system. However, this degradation is drastically reduced to only $26 \%$ for a triple-hop $(K=2)$ relay-assisted QKD system.

Although the triple-hop relay-assisted QKD system can significantly improve the QBER performance, it cannot achieve the QBER performance of the direct non-turbulent case as it might be expected from Fig. 2. This is as a result of the accumulation

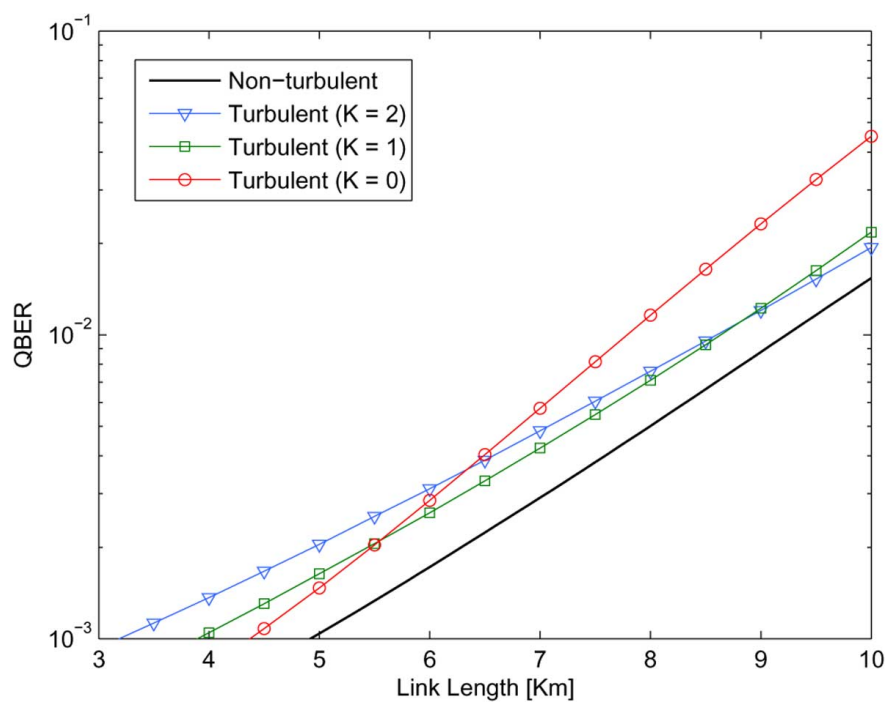

Fig. 3. QBER of the relay-assisted QKD system for different number of relays $\left(d=10 \mathrm{~cm}, n_{s}=1\right)$.

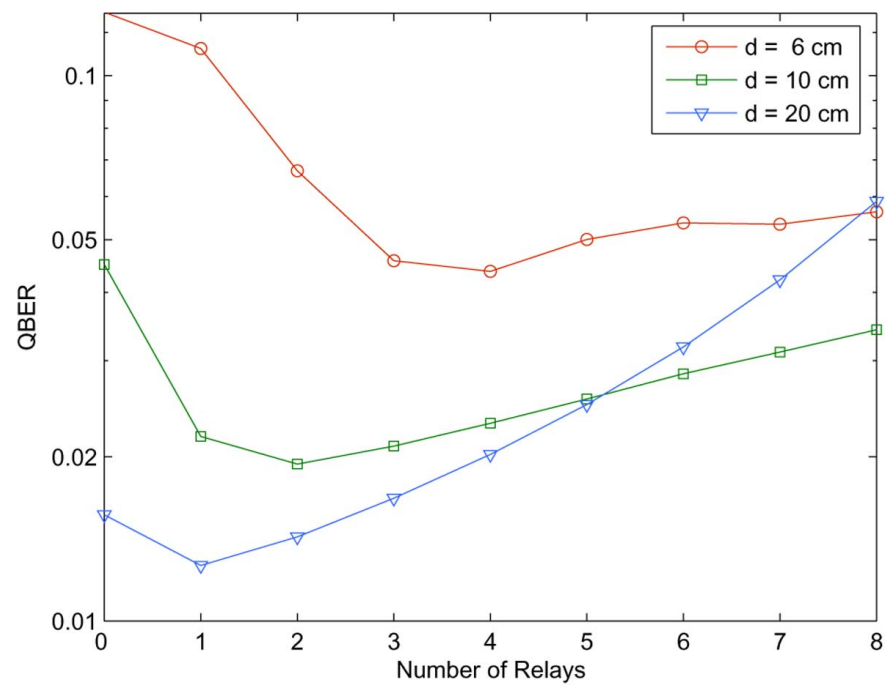

Fig. 4. QBER of the relay-assisted QKD system versus the number of relays with different pupil diameters $\left(L=10 \mathrm{~km}, n_{s}=1\right)$.

of background noise redirected from relays to Bob's receiver. ${ }^{6}$ In other words, there are two reverse effects which need to be considered: the relay-assisted scheme increases the average number of collected photons coming from Alice, but it also results in an increase of the average number of background photons at Bob's receiver. It is therefore important to determine the optimum number of relays (in the sense of QBER minimization) to be deployed.

In Fig. 4, we demonstrate the upper bound on QBER of the relay-assisted QKD system with $n_{s}=1$ versus the number of relays. We fix the link length as $10 \mathrm{~km}$ and consider different pupil diameters i.e., $d=6 \mathrm{~cm}, 10 \mathrm{~cm}$, and $20 \mathrm{~cm}$. We find out that four, two and one relays are sufficient to minimize QBER, respectively, for $d=6 \mathrm{~cm}, 10 \mathrm{~cm}$, and $20 \mathrm{~cm}$. It is observed that the optimum number of relays increases as the pupil size decreases. This comes from the fact that for the system with

${ }^{6}$ Note that, in our analysis, this effect has been exaggerated in derivation of upper bound on QBER of the relay-assisted system, cf. (5) and (23). 


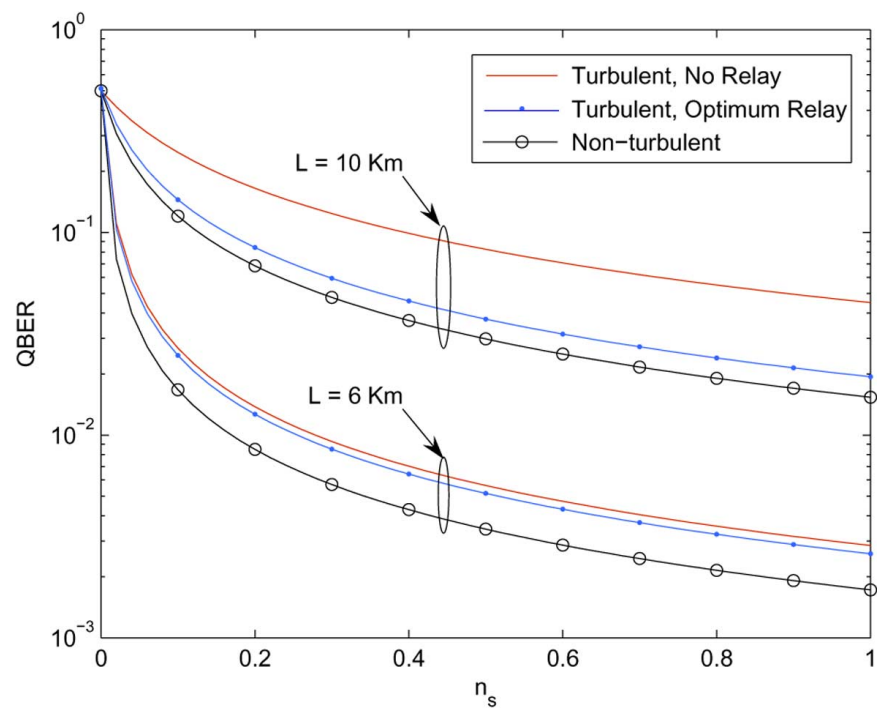

Fig. 5. QBER of the relay-assisted QKD system versus $n_{s}$ for different link lengths $(d=10 \mathrm{~cm})$.

smaller pupil sizes, shorter hops are required for effective compensation of the diffraction and turbulence effects.

In Fig. 5, we demonstrate the upper bound on QBER of the relay-assisted QKD system as a function of the average number of transmitted photons by Alice, $n_{s}$. We consider $L=6 \mathrm{~km}$ and $10 \mathrm{~km}$ and assume $d=10 \mathrm{~cm}$. Note that the QBER performance improvements demonstrated in Figs. 3 and 4 were obtained for a fixed value of $n_{s}=1$. From Fig. 5, we observe that decreasing the value of $n_{s}$ does not considerably affect the amount of QBER improvement and, therefore, similar conclusions can be also made for these lower values of $n_{s}$.

As a final remark on our performance results, we need to re-emphasize that adaptive optics is required to reconstruct the turbulence-degraded received wave-front in the proposed system. Different adaptive optics systems with fully [21]-[23] and partially [24], [25] compensation can be used for this purpose. In a fully compensated adaptive optics system, the scale of the operating elements (sub-aperture of the wave-front sensor and the actuator of the deformable mirror) matches the scale of the atmospheric correlation length which is inversely related to the link length [23], [24]. Since the proposed relay-assisted system divides the link range into shorter hops, adaptive optics can be implemented relatively easier with larger, therefore fewer, elements. Furthermore, we note that the atmospheric coherence time is inversely related to the link length [26]. Therefore, longer atmospheric coherence time can be experienced in the shorter hops decreasing the rate of required updates in the adaptive optics system.

\section{CONCLUSION}

In this paper, we proposed a terrestrial relay-assisted freespace QKD system for long links operating over turbulent atmosphere. Based on a near-field analysis, we derived an upper bound on QBER of the proposed system and presented extensive numerical results on the performance as a function of various system parameters including the number of relays, link range, pupil diameter, etc. Although the relay-assisted scheme results in an increase of the average number of background photons collected at Bob's receiver, it is able to significantly decrease the photon loss caused by diffraction and turbulence. The net gain is an overall performance improvement particularly for long link ranges.

\section{REFERENCES}

[1] G. Van Assche, Quantum Cryptography and Secret-Key Distillation. Cambridge, U.K.: Cambridge Univ. Press, 2006.

[2] C. H. Bennett and G. Brassard, "Quantum cryptography: Public key distribution and coin tossing," in Proc. IEEE Int. Conf. Comput. Syst. Signal Process., Bangalore, India, 1984, pp. 175-179.

[3] A. K. Ekert, "Quantum cryptography based on Bell's theorem," Phys. Rev. Lett., vol. 67, pp. 661-663, 1991.

[4] N. Gisin, G. Ribordy, W. Tittel, and H. Zbinden, "Quantum cryptography," Rev. Mod. Phys., vol. 74, pp. 145-145, 2002.

[5] D. Kedar and S. Arnon, "Urban optical wireless communication networks: The main challenges and possible solutions," IEEE Commun. Mag., vol. 42, no. 5, pp. s2-s7, May 2004.

[6] T. A. Tsiftsis, H. G. Sandalidis, G. K. Karagiannidis, and N. C. Sagias, "Multihop free-space optical communications over strong turbulence channels," in Proc. IEEE Int. Conf. Commun. 2006 (ICC'06), Istanbul, Turkey, Jun. 2006, pp. 2755-2759.

[7] G. K. Karagiannidis, T. A. Tsiftsis, and H. G. Sandalidis, "Outage probability of relayed free space optical communication systems," Electron. Lett., vol. 42, pp. 994-995, Aug. 2006.

[8] M. Safari and M. Uysal, "Relay-assisted free-space optical communication," IEEE Trans. Wireless Commun., vol. 7, no. 12, pp. 5441-5449, Dec. 2008.

[9] J. H. Shapiro, "Near-field turbulence effects on quantum-key distribution," Phys. Rev. A, vol. 67, no. 2, pp. 022309-022309, 2003.

[10] M. Gabbi and S. Arnon, "Quantum key distribution by free space MIMO system,” J. Lightw. Technol., vol. 24, no. 8, pp. 3114-3140, Aug. 2006.

[11] G. Gilbert and M. Hamrick, "Practical quantum cryptography: A comprehensive analysis (Part One)," MITRE Tech. Rep., 2000, preprint quant-ph/0009027.

[12] R. J. Hughes, J. E. Nordholt, D. Derkacs, and C. G. Peterson, "Practical free-space quantum key distribution over $10 \mathrm{~km}$ in daylight and at night," New J. Phys., vol. 4, pp. 43-43, 2002.

[13] M. Gabby and S. Arnon, "The effect of turbulence on a quantum-key distribution scheme based on transformation from the polarization to the time domain: Laboratory experiment," Opt. Eng., vol. 44, no. 4, pp. 045002-10-045002-6, Apr. 2005.

[14] K. J. Resch, M. Lindenthal, B. Blauensteiner, H. R. Böhm, A. Fedrizzi, C. Kurtsiefer, A. Poppe, T. Schmitt-Manderbach, M. Taraba, R. Ursin, P. Walther, H. Weier, H. Weinfurter, and A. Zeilinger, "Distributing entanglement and single photons through an intra-city, free-space quantum channel," Opt. Exp., vol. 13, pp. 202-209, 2005.

[15] H. J. Briegel, W. Dür, J. I. Cirac, and P. Zoller, "Quantum repeaters: The role of imperfect local operations in quantum communication," Phys. Rev. Lett., vol. 81, pp. 5932-5935, 1998.

[16] B. C. Jacobs, T. B. Pittman, and J. D. Franson, "Quantum relays and noise suppression using linear optics," Phys. Rev. A, vol. 66, pp. 052307-052307, 2002.

[17] C. Elliot, "Building the quantum network," New J. Phys., vol. 4, pp. 46.1-46.12, 2002.

[18] H. Bechmann-Pasquinucci and A. Pasquinucci, "Quantum key distribution with trusted quantum relay," 2005, eprint quant-ph /0505089.

[19] R. J. Hughes, W. T. Buttler, P. G. Kwiat, S. K. Lamoreaux, G. L. Morgan, J. E. Nordholt, and C. G. Peterson, "Free-space quantum key distribution in daylight," J. Mod. Opt., vol. 47, pp. 549-562, 2000.

[20] M. Aspelmeyer, T. Jennewein, M. Pfenningbauer, W. R. Leeb, and A. Zeilinger, "Long-distance quantum communication with entangled photons using satellites," IEEE J. Sel. Topics Quantum Electron., vol. 9, pp. 1541-1551, 2003.

[21] J. W. Hardy, J. E. Lefebvre, and C. L. Koliopoulos, "Real-time atmospheric compensation," J. Opt. Soc. Amer., vol. 67, pp. 360-369, 1976.

[22] J. R. P. Angel, "Ground-based imaging of extrasolar planets using adaptive optics," Nature, vol. 368, pp. 203-207, 1994.

[23] B. M. Levine, E. A. Martinsen, A. Wirth, A. Jankevics, M. ToledoQuinones, F. Landers, and T. L. Bruno, "Horizontal line-of-sight turbulence over near-ground paths and implications for adaptive optics corrections in laser communications," Appl. Opt., vol. 37, pp. 4553-4560, 1998. 
[24] P. Nisenson and R. Barakat, "Partial atmospheric correction with adaptive optics," J. Opt. Soc. Amer. A, vol. 4, pp. 2249-2253, 1987.

[25] M. C. Roggemann, "Limited degree-of-freedom adaptive optics and image reconstruction," Appl. Opt., vol. 30, pp. 4227-4233, 1991.

[26] L. C. Andrews, R. L. Phillips, and C. Y. Hopen, Laser Beam Scintillation With Applications. Bellingham, WA: SPIE Press, 2001.

[27] J. H. Shapiro, "Normal-mode approach to wave propagation in the turbulent atmosphere," Appl. Opt., vol. 13, pp. 2614-2614, 1974.

[28] D. Slepian, "Analytic solution of two apodization problems," J. Opt. Soc. Amer., vol. 55, pp. 1110-1115, 1965.

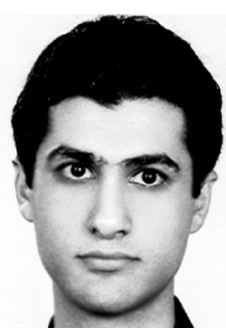

Majid Safari (S'08) received B.Sc. degree in electrical engineering from the University of Tehran, Tehran, Iran, in 2003 and M.Sc. degree in electrical engineering from Sharif University of Technology, Tehran, in 2005. He is currently pursuing the Ph.D. degree in electrical engineering at the University of Waterloo, Waterloo, ON, Canada.

His research interests include free-space optical communications and optical fiber communications.

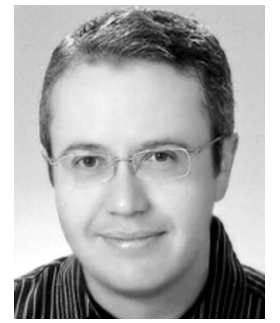

Murat Uysal (M'02-SM'07) was born in Istanbul, Turkey, in 1973. He received the B.Sc. and the M.Sc. degrees in electronics and communication engineering from Istanbul Technical University, Istanbul, Turkey, in 1995 and 1998, respectively, and the $\mathrm{Ph} . \mathrm{D}$. degree in electrical engineering from Texas A\&M University, College Station, TX, in 2001.

Since 2002, he has been with the Department of Electrical and Computer Engineering, University of Waterloo, Waterloo, ON, Canada, where he is now an Associate Professor. His general research interests lie in communications theory and signal processing for communications with special emphasis on wireless applications. Specific research areas include MIMO techniques, space-time coding, diversity techniques and coding for fading channels, cooperative communications, and free-space optical communications.

Dr. Uysal is an Associate Editor for IEEE TRANSACTIONS ON WIRELESS CommunicAtions and IEEE COMmunications LetTers. He was a Guest Co-Editor for Wiley Journal on Wireless Communications and Mobile Computing's Special Issue on "MIMO Communications" published in 2004. He is currently serving as a Guest Co-Editor for the IEEE JOURNAL ON SELECTED AREAS IN COMMUNICATIONS' Special Issue on "Optical Wireless Communications" to be published in 2010. Over the years, he has served on the technical program committee of more than 50 international conferences in the communications area. He recently co-chaired IEEE ICC' 07 Communication Theory Symposium and CCECE'08 Communications and Networking Symposium. 